\title{
Br-SCMM: Modelo Brasileiro de Maturidade para Cidades Inteligentes
}

\author{
Ricardo Alexandre Afonso ${ }^{1,2}$, Welington M. da Silva ${ }^{1,3}$, Gustavo H. R. P. Tomas ${ }^{1,3}$, \\ Kiev Gama ${ }^{1}$, Alezy Oliveira ${ }^{1,2}$, Alexandre Alvaro ${ }^{1,3}$, Vinicius Cardoso Garcia ${ }^{1}$ \\ ${ }^{1}$ Universidade Federal de Pernambuco (UFPE) - Centro de Informática (CIn) \\ Recife - Pernambuco - Brasil \\ ${ }^{2}$ Universidade Federal de Alagoas (UFAL) - Computação (AraComp) \\ Arapiraca - Alagoas - Brasil \\ ${ }^{3}$ Universidade Federal de São Carlos (UFSCar) - Depto. de Computação (DComp) \\ Sorocaba - SP - Brasil \\ \{raa4, wms4, ghrpt, kiev, aa, vcg\}@cin.ufpe.br
}

\begin{abstract}
The Smart Cities are emerging ahead of the needs of the new society and information technology that has emerged in this century. Currently there is a maturity model that can measure how intelligent a society can be based on social or technological indicators. Create a maturity model assumes establish requirements, analyze them and determine which will be transformed into indicators. This paper presents the initial level of a maturity model applied to intelligent cities.
\end{abstract}

Resumo. As Cidades Inteligentes estão emergindo frente às necessidades da nova sociedade da tecnologia e informação que surgiu neste século. Atualmente não existe um modelo de maturidade que possa medir o quão inteligente uma sociedade pode ser com base em indicadores sociais ou tecnológicos. Criar um modelo de maturidade pressupõe levantar requisitos, analisá-los e definir quais serão transformados em indicadores. Este trabalho visa apresentar o nível inicial de um Modelo de maturidade aplicado às cidades inteligentes.

\section{Introdução}

Segundo estimativas das Nações Unidas (ONU), o número de habitantes dos centros urbanos vai alcançar mais de cinco bilhões ate o ano de 2030. Atualmente, as cinco maiores cidades do mundo são mais populosas do que vários países no globo. Conforme o relatório elaborado pela empresa de consultoria PriceWaterHouseCoopers [PRICE 2009] que contém um ranking com as 100 cidades e/ou suas áreas metropolitanas mais ricas do mundo pelo PIB, o Brasil possui cinco lugares garantidos, através das cidades de: São Paulo, Rio de Janeiro, Brasília, Porto Alegre e Belo Horizonte ocupando respectivamente as posições: 10, 31, 57, 89 e 91 .

A forma como são gerenciadas estas cidades pode servir de modelo para as cidades de menor porte, que poderão no futuro se beneficiar dos resultados de administrações bem sucedidas. Para que se obtenham esses resultados, se 
faz necessário medir o quão bem sucedida uma cidade está sendo administrada sob os mais diversos domínios, e entre eles, a Água, Saúde e Educação figuram como os mais básicos e importantes domínios citados nos estudos sobre Cidades Inteligentes.

A principal motivação deste trabalho é criar um modelo de maturidade capaz de incentivar cidades brasileiras a identificarem seus potenciais e melhorarem seus processos e políticas públicas, otimizando assim seus recursos. Para isso, a seção 2 deste trabalho apresenta uma visita aos trabalhos prévios que exploram a definição do termo "Cidade Inteligente", seus domínios e indicadores. A seção 3 apresenta os indicadores básicos que compõe o primeiro nível do modelo de maturidade proposto. Fica a cargo da seção 4 apresentar o modelo de maturidade enquanto a seção 5 traz os resultados de sua utilização nas capitais brasileiras. A seção 6 conclui este trabalho com as considerações finais.

\section{Cidades Inteligentes: Trabalhos prévios}

$\mathrm{Na}$ busca pela definição do conceito de Cidades Inteligentes, seus domínios e áreas, vários autores revisitaram a bibliografia sobre o assunto e alguns propõem indicadores necessários para compor essa definição.

Segundo [Van Den Berg 1999], o conceito de inteligência quando aplicado a sistemas, organizações ou regiões está diretamente interligado com as competências associadas à análise da informação.

No ano de 2002 [Komninos, 2002] utilizou pela primeira vez o conceito de Cidades e Regiões Inteligentes. Segundo a definição do autor, as Cidades e Regiões Inteligentes: Combinam o ambiente digital e comunidades reais; Possuem elevado nível de conhecimento; Pertencem a uma área geográfica que partilha o conhecimento; Dependem de uma infraestrutura baseada em tecnologias de informação e comunicação e Otimizam a gestão do conhecimento.

O Centre of Regional Science (2007) elaborou um ranking onde aponta 70 cidades europeias de médio porte que atendem a requisitos indicados em seu modelo de Cidade Inteligente. Este modelo se divide em seis características compostos por conjuntos de fatores que detalham a capacidade de Competitividade, os aspectos sociais, a participação da população na tomada de decisões, qualidade de vida, transporte e recursos humanos.

Iniciativas comerciais lideradas pela IBM [IBM 2009] apostam no desenvolvimento de soluções que se baseiam em Infraestrutura, Operações e Pessoas. Dessa forma, essa soluções utilizam uma divisão de 9 fatores para definir a estratégia de implementação e utilização de Cidades inteligentes.

A seção seguinte apresenta os domínios e indicadores que serão utilizados para balizar a construção do modelo de maturidade em seu nível inicial.

\section{Domínios e indicadores básicos.}

Para criar a relação dos indicadores básicos, foram considerados dois fatores importantes: A disponibilidade de dados para medição e a possibilidade de comparação desses dados locais com o mesmo conjunto de dados levantados em outros municípios do Brasil e do mundo. Este trabalho utilizou a extração e catalogação manual os dados 
disponibilizados em bases de dados públicas, e os domínios mensurados foram: Água, Saúde e Educação. Esta seção detalha alguns aspectos desses indicadores em cidades brasileiras.

\subsection{Domínio Água (Indicador: Água encanada)}

O Domínio Água foi apontado nos trabalhos de [Deakin, Caraglio 2007] como fundamental para definição de Cidades Inteligentes. Uma forma bastante elementar de avaliar a qualidade do serviço de água nos municípios é através do indicador do percentual de residências atendidas por água encanada.

Segundo o IBGE$^{1}$, entre os 5570 municípios brasileiros, apenas três possuem todas as residências abastecidas por água encanada e saneamento enquanto 2147 municípios obtiveram um índice igual ou superior a 90\% de abastecimento de água residencial. Foram utilizados os dados dos portais $\mathrm{IPEA}^{2}$ e $\mathrm{ODM}^{3}$ para levantar os dados referentes ao serviço publico de água e esgoto nos municípios brasileiros.

\subsection{Domínio Saúde (Indicador: IDH)}

Atualmente o DataSus ${ }^{4}$ é responsável por aferir os dados referentes ao atendimento básico de saúde no pais. E a organização TrataBrasil ${ }^{5}$ realiza um trabalho de acompanhamento destes atendimentos e do nível de satisfação dos usuários do serviço publico de saúde.

Para calcular o indicador que represente a saúde de uma cidade, foi utilizado como indicador o IDH (Índice do Desenvolvimento Humano). Este índice foi remodelado em 2010 e passou a usar um novo método de cálculo que se baseia na expectativa de vida, no índice de educação e índice de renda.

\subsection{Domínio Educação (Indicador: IDEB)}

Sob o ponto de vista social, a Educação pode ser vista como o domínio responsável pelo incremento e influência direta em vários outros domínios, pois, na medida em que uma sociedade se torna mais educada, ela também se torna mais saudável e segura.Este conceito também pode ser encontrado nos trabalhos de [Malek, 2009 e Borja, 2007], que apontam a educação como um dos domínios presentes na definição de cidades inteligentes.

No Brasil, o $\mathrm{MEC}^{6}$ (Ministério da Educação) utiliza o IDEB (Índice de Educação Básica) para fornecer dados sobre a qualidade da educação básica. O índice é medido a cada dois anos e o objetivo é que o país, a partir do alcance das metas municipais e estaduais, consiga atingir uma nota igual a 6 , que corresponde à qualidade do ensino básico em países desenvolvidos.

A próxima seção apresenta a concepção do modelo de maturidade, seus domínios e indicadores.

\footnotetext{
${ }^{1}$ IBGE, Instituto Brasileiro de Geografia e Estatística, disponível em: http://www.ibge.gov.br

${ }^{2}$ IPEA, Instituto de Pesquisa Econômica Aplicada, disponível em: http://www.ipea.gov.br

${ }^{3}$ ODM, Sistema de Indicadores municipais on-line, disponível em: http://www.portalodm.com.br

${ }^{4}$ DATASUS, Dados do Sistema Único de Saúde, disponível em: http://www.datasus.gov.br

${ }^{5}$ TRATABRASIL, Saneamento é saúde, disponível em: http://www.tratabrasil.org.br

${ }^{6}$ MEC, Ministério da educação, disponível em: http://emec.mec.gov.br
} 


\section{Modelo de Maturidade para Cidades Inteligentes}

Modelos de Maturidade geralmente tem como proposito fazer com que organizações possam mensurar e otimizar a progressão de capacidades e competências desenvolvidas em determinada área.

$\mathrm{Na}$ área de engenharia de software, o modelo de maturidade CMMI (Capability Maturity Model Integration) utiliza práticas genéricas e especificas e foi desenvolvido para padronizar e mensurar a qualidade do processo de melhoria corporativo, integrando diferentes modelos e disciplinas. Já o MPS.BR ou Melhoria de Processos do Software Brasileiro é um modelo de maturidade utilizado para ampliar a qualidade de processo de produção de sistemas ao mesmo tempo em que atende a realidade do mercado, além de ser compatível com o CMMi.

O trabalho proposto em [Gama, 2012] aponta o uso de um modelo de maturidade voltado à tecnologia para cidades inteligentes. Nesse trabalho utilizamos indicadores relacionados à tecnologia para compor um dos domínios a ser mensurado.

O modelo proposto nesse trabalho chamado Br-SCMM (Brazilian Smart Cities Maturity Model), - Modelo Brasileiro de Maturidade para Cidades Inteligentes - utiliza os domínios de Água, Saúde e Educação para mensurar o primeiro nível em uma escala que determina o quão inteligente uma cidade pode se tornar. Este modelo se divide em cinco níveis não incrementais, de forma que um município possa evoluir nos domínios em que tiver maior aptidão e recursos. Estes níveis são divididos em cinco categorias e compõe a palavra (SMART), visando responder a cinco questões sobre Cidades Inteligentes:

- Nível S: (simplified): Simplificado: A cidade alcança notas mínimas nos indicadores chamados de indicadores básicos?

- Nível M: (Managed): Gerenciado: A cidade possui objetivos e práticas que apontem para um gerenciamento otimizado dos recursos?

- Nível A: (Applied): Aplicado: A cidade utiliza o modelo de maturidade para estabelecer suas politicas públicas?

- Nível R: (Rated): Medido: A cidade estabeleceu indicadores e possui práticas de medição e melhoria de desempenho?

- Nível T: (Turned): Transformado: A cidade atingiu notas desejadas para os domínios nos quais aplica suas praticas especificas e gerais?

A Seção seguinte apresenta os resultados obtidos com utilização deste modelo em seu primeiro nível.

\section{Resultados}

Foram obtidos dados das vinte e sete capitais brasileiras sobre os domínios e indicadores especificados na seção 3 deste trabalho. Esses dados passaram por normalização matemática e foram adequados a uma escala de zero a cinco. A Figura 1 apresenta a comparação entre as capitais brasileiras e seus indicadores em relação ao nível inicial do modelo. 


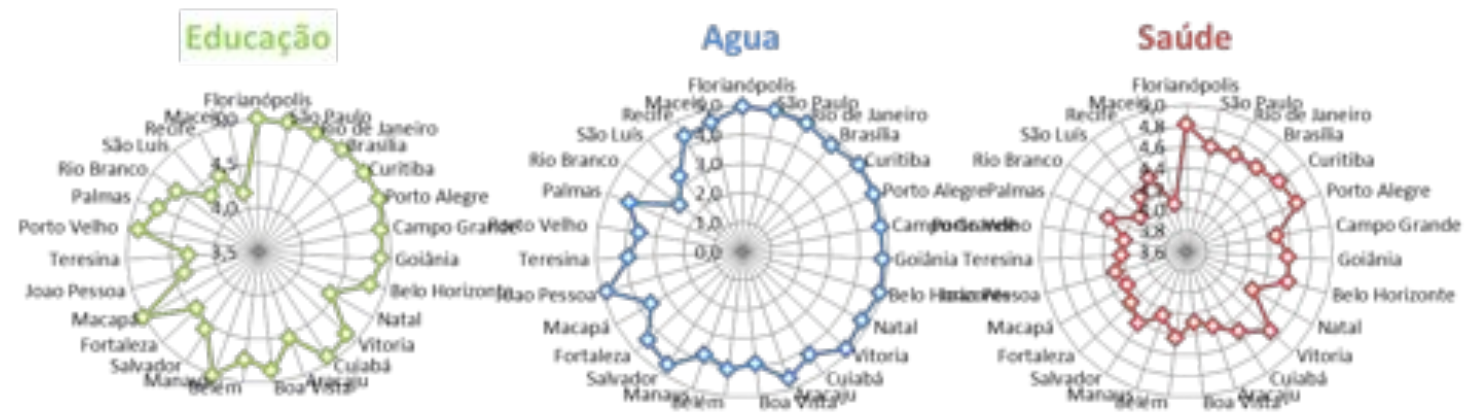

Figura 1. SCMM: Comparação de Domínios entre Capitais Brasileiras.

Desta forma, analisando o nível inicial deste modelo de maturidade é possível indicar quais cidades necessitam ajustar suas práticas especificas e gerais para alcançar melhores notas nos domínios desejados e se for do desejo dessas cidades, buscar índices mais próximos das demais capitais.

São apontadas na Figura 2 os dez maiores municípios brasileiros em número populacional, e sua relação com o indicador "Educação". O resultado pode ser mensurado pelas médias e pela média linear. Essa informação permite adequar os dados ao modelo, e assim estabelecer os objetivos e práticas a seguir.

\begin{tabular}{|l|c|c|c|c|}
\hline Municípios & Educação & Água & Saúde & Média \\
\hline São Paulo & 5,0 & 4,9 & 4,6 & $\mathbf{4 , 8 5}$ \\
\hline Rio de Janeiro & 5,0 & 4,9 & 4,6 & $\mathbf{4 , 8 4}$ \\
\hline Brasília & 5,0 & 4,7 & 4,6 & $\mathbf{4 , 7 9}$ \\
\hline Curitiba & 5,0 & 5,0 & 4,7 & $\mathbf{4 , 8 9}$ \\
\hline Porto Alegre & 5,0 & 4,9 & 4,8 & $\mathbf{4 , 8 8}$ \\
\hline Belo Horizonte & 4,8 & 4,9 & 4,6 & $\mathbf{4 , 7 8}$ \\
\hline Manaus & 5,0 & 3,8 & 4,3 & $\mathbf{4 , 3 4}$ \\
\hline Salvador & 4,6 & 4,7 & 4,4 & $\mathbf{4 , 5 5}$ \\
\hline Fortaleza & 4,4 & 4,4 & 4,3 & $\mathbf{4 , 4 0}$ \\
\hline Recife & 4,5 & 4,4 & 4,4 & $\mathbf{4 , 4 1}$ \\
\hline \multicolumn{1}{|c|}{ Média } & $\mathbf{4 , 8 3}$ & $\mathbf{4 , 6 5}$ & $\mathbf{4 , 5 4}$ & \multicolumn{4}{|l}{} \\
\cline { 1 - 3 }
\end{tabular}

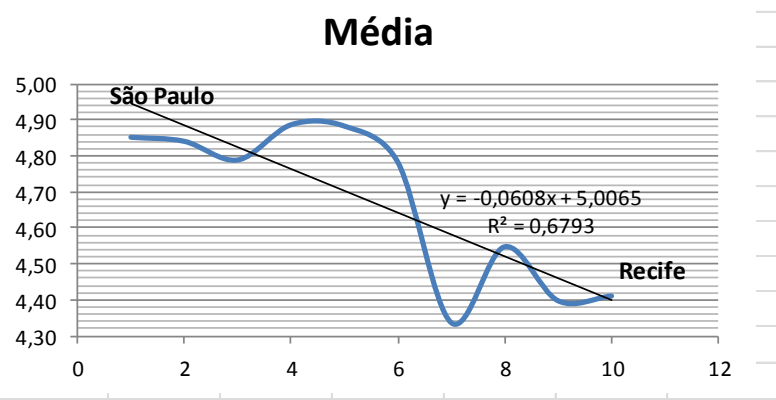

Figura 2. SCMM: Nível 1 do modelo entre os municípios mais populosos.

A função do nível inicial (Nível S) não é impedir que as cidades pudessem avançar para os níveis seguintes, mas sim, fornecer uma perspectiva que possibilite os ajustes necessários dos indicadores básicos. Ao melhorar os índices básicos, o município pode almejar notas melhores no nível seguinte (Nível M)

\section{Considerações finais.}

Este trabalho apresentou os resultados obtidos com a coleta, normalização e comparação de dados entre as capitais brasileiras. O modelo de Maturidade composto por cinco níveis está sendo desenvolvido e testado junto as capitais brasileiras nível a nível. Atualmente o modelo conta com dados coletados em dez diferentes domínios: Educação, Saúde, Água, Energia, Governança, Segurança, Meio Ambiente, Habitação e Tecnologia.

Durante os próximos anos a otimização e gerenciamento de recursos será um dos maiores desafios a serem encarados no mundo aberto. Para isso, os municípios poderão contar com um modelo de maturidade brasileiro, extensível e adaptativo a realidade na qual estamos inseridos. 
Uma importante contribuição para os trabalhos futuros será o desenvolvimento de processos de extração e catalogação automática desses repositórios de dados, que hoje é feita manualmente. Transformar essas bases de dados em Open Linked Data já faz parte de uma etapa em andamento deste projeto.

\section{Agradecimentos}

Este trabalho foi parcialmente financiado pelo Instituto Nacional de Ciência e Tecnologia para Engenharia de Software (INES) e financiado pelo CNPq, FAPESP, 2012/10157-1, e FACEPE, bolsas 573964/2008-4, APQ-1037-1.03/08. Ricardo A. Afonso é parcialmente financiado pela FACEPE, concessão IBPG-0499-1.03/11.

\section{Referências}

PRICE (2009) Which are the largest city economies? http://www.ukmediacentre.pwc.com/imagelibrary/downloadMedia.ashx?MediaDetail sID=1562, 2009. "[Online] Available: 27-Januaryt-2013"

Van Den Berg, L. e Braun, E. (1999) Urban Competitiveness, Marketing and The Need for Organising Capacity. Urban Studies, 36(5-6), 987-999

Komminos, N (2002). Intelligent Cities: Innovation, Knowledge Systems and Digital Spaces. London: Routledge, 2002.

Centre of Regional Science (2007)."Smart cities - Ranking of European medium-sized cities". http://www.smart-cities.eu/. Vienna. "[Online] Available: 27-Januaryt-2013"

IBM Institute for Business Value, (2009). A Vision of Smarter Cities- How Cities Can Lead the Way into a Prosperous and Sustainable Future, IBM Global Business Services Executive Report, 2009.

Deakin, M; Allwinkle, S (2007). "Urban regeneration and sustainable communities: the role networks, innovation and creativity in building successful partnerships". Journal of Urban Technology 14 (1): 77-91.

Caragliu, A; Del Bo, C. \& Nijkamp, P (2007). "Smart cities in Europe". Serie Research Memoranda 0048 (VU University Amsterdam, Faculty of Economics, Business Administration and Econometrics).

Malek, J. A. (2009). Informative global community development index of informative smart city. In Proceedings of the 8th WSEAS International Conference on Education and Educational Technology(Genova, Italy, Oct 17-19).

Borja, J. (2007). Counterpoint: Intelligent cities and innovative cities. Universitat Oberta de Catalunya (UOC) Papers: E-Journal on the Knowledge Society, 5. Available at http://www.uoc.edu/uocpapers/5/dt/eng/mitchell.pdf. "[Online] Available: 27Januaryt-2013"

GAMA, K. ; ALVARO, A. ; PEIXOTO, E. . Em direção a um modelo de maturidade tecnológica para cidades inteligentes. In: Simpósio Brasileiro de Sistemas de Informação, 2012. Anais do VIII Simpósio Brasileiro de Sistemas de Informação, 2012. p. 150-155. 